\title{
El Dr. Felipe Alfonso Aceves Zubieta, formador de médicos y medios de comunicación
}

\section{Dr. Felipe Alfonso Aceves Zubieta, trainer of physicians and media}

\author{
Dr. Rolando Neri-Vela,* Dr. Carlos Agustín Rodríguez-Paz, ${ }^{\ddagger}$ \\ Dr. Luis Sánchez-Fernández, ${ }^{\S}$ Primer Mtre. SSN IP. Ericka Medina-González
}

Citar como: Neri-Vela R, Rodríguez-Paz CA, Sánchez-Fernández L, Medina-González E. El Dr. Felipe Alfonso Aceves Zubieta, formador de médicos y medios de comunicación. Rev Mex Anestesiol. 2021; 44 (2): 148-150. https://dx.doi.org/10.35366/99020

\section{RESUMEN}

Felipe Alfonso Aceves Zubieta (1899-1960) fue un médico mexicano del siglo XX, profesor de varias asignaturas quirúrgicas en la Escuela Nacional de Medicina, y fundador de las Asambleas Nacionales de Cirujanos (1934), de la Revista de Cirugía del Hospital Juárez y de la Academia Mexicana de Cirugía.

\section{ABSTRACT}

Felipe Alfonso Aceves-Zubieta (1899-1960), was a Mexican physician, who teached several surgical subjects at National School of Medicine, in Mexico City, and was the founder of Asambleas Nacionales de Cirujanos (1934), Revista de Cirugía del Hospital Juárez and Academia Mexicana de Cirugía.

$F_{1}$ elipe Alfonso Aceves Zubieta (Figura 1) nació en la ciudad de México el 09 de agosto de 1899 y falleció en la misma el 22 de junio de 1960.

Realizó sus estudios en la Escuela Nacional de Medicina donde se desempeñó durante años como profesor. Su título profesional fue expedido por la Universidad Nacional Autónoma de México en 1928 con el número 761DSP y el 2115 de la Dirección General de Profesiones, siendo su tesis recepcional Contribuciones al estudio de los aneurismas traumáticos.

En su hoja de servicios de la escuela el Dr. Aceves Zubieta dio a conocer su domicilio particular en el número 5 de la calle Comunal en Villa Obregón, y el de su consultorio en la calle República de Cuba número 75, despachos 303, 304 y 305, en el actual Centro Histórico de la Ciudad de México, lo que nos muestra que era un médico exitoso ${ }^{(1)}$.

Habiendo laborado en el Hospital Juárez, fue jefe de clínica quirúrgica hacia 1925. El Dr. Mendizábal-Guerra lo considera dentro de los cirujanos que iniciaron la neurocirugía en el Hospital Juárez ${ }^{(2)}$.

En la Escuela Nacional de Medicina fue ayudante de clínica en 1926; en 1930 profesor interino en varias asignaturas; en 1931 profesor adjunto del Tercer Curso de Clínica Quirúrgica; en 1934 profesor del Segundo Curso de Clínica Quirúrgica; en 1935 profesor del Segundo Curso de Clínica Quirúrgica en el Quinto año de medicina; en 1939 profesor titular conferencista especializado del Tercer Curso de Clínica Quirúrgica; en 1947 profesor ordinario de la Cátedra del Tercer Curso de Patología Quirúrgica, entre otros más, hasta que causó baja en la universidad por defunción ${ }^{(3)}$.

El doctor Aceves Zubieta fue miembro del Círculo Médico Católico, en el que fue bien reconocido. Dicha asociación, fundada en 1953 por monseñor Gastón Mijaisky Perrelli, tenía a Aceves Zubieta como su presidente en 1958. Ese mismo año, con motivo de la décima tercera Asamblea Nacional de Cirujanos el Círculo Médico Católico publicó el libro Actualización

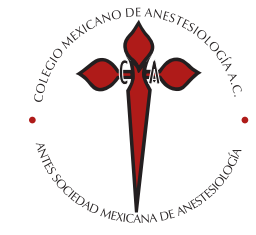

Palabras clave: Medicina, cirugía, enseñanza.

Keywords:

Medicine, surgery, teaching.

* Escuela Médico Naval.

Comisión de Estudios Históricos

Escuela Médico Militar.

‡ Escuela de Medicina.

Universidad Cuauhtémoc. Plantel

San Luis Potosí, Servicio de

Cirugía. Hospital 50 IMSS.

$\S$ Facultad de Medicina.

Universidad de Oviedo. España.

" Escuela Médico Naval,

Universidad Naval. Secretaría de

Marina-Armada de México.

Correspondencia:

Dr. Rolando Neri-Vela

Tuxpan No. 16-401,

Col. Roma Sur, CDMX,

06760, México

E-mail: drnerivela@hotmail.com

Recibido: 29-01-2021

Aceptado:03-02-2021 


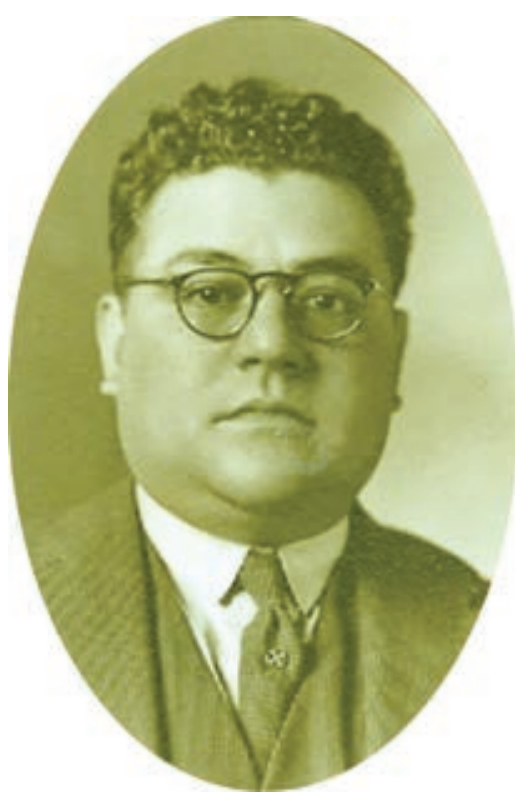

Figura 1:

Dr. Felipe Alfonso Aceves Zubieta $^{(4)}$.

deontológica de la profesión de médico cirujano, y Aceves Zubieta escribió para éste Deontología del cirujano general; la obra fue distribuida entre los médicos que concurrieron a la reunión por cortesía de los Laboratorios Lepetit de México, SA ${ }^{(5)}$.

De acuerdo al expediente del Archivo Histórico de la Facultad de Medicina de la Universidad Nacional Autónoma de México, el 02 de septiembre de 1943 el licenciado Alfredo B. Cuéllar, recién nombrado por el Consejo Consultivo del Distrito Federal como consejero propietario, representante de los profesionistas, envió al director de la Escuela Nacional de Medicina, Gustavo Argil, una carta para que se nombrara a algún representante de esa escuela, por lo que Felipe Alfonso Aceves Zubieta fue nominado para tal encargo, suplicándole que se entrevistara e hiciera de su conocimiento aquellos puntos que interesaran a los profesores y médicos de la facultad para ser defendidos ante las autoridades del Distrito Federal.

A su muerte el 22 de junio de 1960, y habiendo sido velado en la Agencia Eusebio Gayosso, cita en la calle Sullivan esquina con Rosas Moreno en la colonia San Rafael, la Academia Mexicana de Cirugía y la Sociedad Mexicana de Anatomía publicaron en un periódico de la capital sendas esquelas en su honor ${ }^{(6)}$.

De vida creativa e inquieta, Aceves Zubieta formó parte de un grupo de médicos, entre los que se contaban José Castro Villagrana, José Torres Torija, José Negrete Herrera y Hernández Manero, que después de algunas reuniones en el Café Tacuba y en la sala 7 del Hospital Juárez, decidieron realizar la Primera Asamblea Nacional de Cirujanos que se llevó a cabo en noviembre de $1934^{(7)}$.

El Dr. Felipe Aceves Zubieta presidió el Comité Organizador de la II Asamblea Nacional de Cirujanos.
En 1929 un grupo progresista de médicos entusiastas que en esa época llegaban de Europa donde habían permanecido temporalmente aprendiendo los avances de la medicina, propusieron durante una de las juntas impulsar y fortalecer la revista del hospital. El Dr. Felipe Aceves Zubieta en la carta editorial de la Revista del Hospital Juárez, editada por médicos de la institución, envió un saludo cordial y caballeroso a todos los órganos de la prensa médica nacional y extranjera, a todos los médicos mexicanos, a la Facultad de Medicina, a los centros educativos, refiriéndose a los hospitales así como a maestros y alumnos: «La Revista del Hospital Juárez -continúa el Dr. Aceves- ve la luz pública gracias al esfuerzo particular de un grupo de médicos del Hospital de San Pablo» ${ }^{(8)}$.

Recordemos que el 19 de agosto de 1847 fue fundado el Hospital de San Pablo en la vieja construcción que había sido convento de agustinos, para instalar en ese sitio un hospital de sangre que sirviera para atender a los heridos durante la lucha en contra de la invasión de los Estados Unidos, y a la muerte del presidente Benito Juárez García en 1872, por acuerdo del Ayuntamiento de la Ciudad de México, el nosocomio pasó a llamarse Hospital Juárez.

Fundada oficialmente la Sociedad de Cirugía, Aceves Zubieta apunta que «en el Hospital Juárez se ha creado la

\section{Primera Asamblea Nacional de Cirujanos. \\ SENOR DOCTOR}
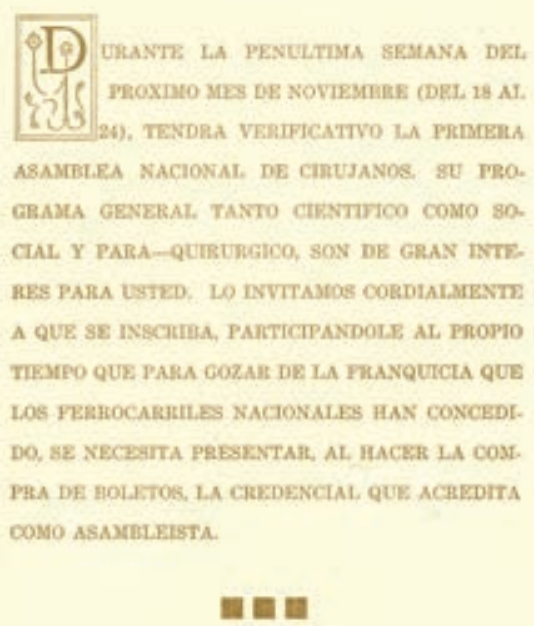

DHEASE UXTED AL SALO, GEXSBAT. DH. VIHE ACEVE MUHETA.

HogetraL JUAkRz.

Figura 2: Contraportada de la revista de la Sociedad de Cirugía ${ }^{(9)}$. 
DIRECTORIO

\section{REVISTA DE CIRUGIA}

Publicación Científica Mensual, editada por médicos del HOSPITAL JUAREZ.

Registrado como artículo de 20 elase, en enero 10 de 1930. Organo de la Sociedad de Cirugia.

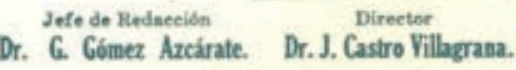

Dres. Jose Castro Villagrana, Felipe Aceves, José Aguilar Alvarez, Gustavo Baz, Clemente Robles y Gustavo Gómez Azeárate

Los originales no se devuelven, aun cuando no hayan sido publicados. Son responsables de las opiniones emitidas y de los articulos, sólo sus autores. Redacción y Administración: Hospital Juárez, Plaza de Sn. Pablo 13 MEXICO, D. F.

Precio: 50 cts. Subscripción anual $\$ 4.00$, en el extranjero $\$ 50.0$

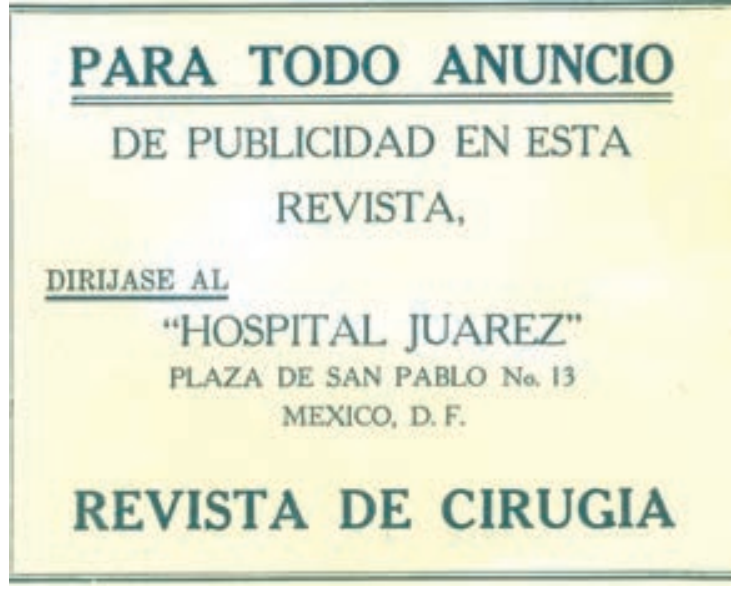

Figura 3:

Segunda de forros de la revista de la Sociedad de Cirugía ${ }^{(9)}$.
Sociedad de Cirugía, cuyo significado en el medio científico y social vale la pena examinar, puesto que su aparición es el resultado del deseo ineludible de mejorar el trabajo personal mediante una colaboración amistosa para obtener el bienestar y el progreso del grupo íntegro» ${ }^{(8)}$. En los años 50 fue director del llamado Centro Médico Universitario en la nueva Ciudad Universitaria de la Universidad Nacional Autónoma de México, haciendo entre otras labores los exámenes médicos de ingreso a los nuevos universitarios ${ }^{(10)}$.

Don Felipe Alfonso Aceves Zubieta fungió como secretario general en la Primera Asamblea Nacional de Cirujanos, y fue miembro del Comité Editorial de la Revista de Cirugía Hospital Juárez (Figuras 2 y 3), siendo uno de los fundadores de la Academia Mexicana de Cirugía.

Dentro de la producción bibliográfica del Dr. Aceves Zubieta destacan los siguientes trabajos publicados en
Cirugía y Cirujanos, órgano de difusión de la Academia Mexicana de Cirugía: El neumoperitoneo antiperiviscerítico (1933), Valor clínico del sufrimiento del plexo solar en el diagnóstico y pronóstico de las afecciones abdominales (1955), Preocupaciones neuroquirúrgicas acerca del cáncer cerebral (1959) y La cara posterior del abdomen desde el punto de vista de la clínica del vientre (1959)(11). También aportó trabajos para la revista Hospital General, Revista Mexicana de Ciencias Médicas como fueron un Nuevo procedimiento de circunsición (sic) (1925), Esplenectomía total (1926) y Sobre la transfusión sanguínea (1927). Además aportó múltiples comentarios en las diversas revistas médicas de su época.

Hombre de ciencia, Felipe Aceves Zubieta será recordado por los médicos mexicanos como un ejemplo de estudio y de perseverancia.

\section{REFERENCIAS}

1. Archivo histórico de la Facultad de Medicina de la Universidad Nacional Autónoma de México (AHFM), vol. 1, exp. 7.

2. Mendizábal-Guerra R. El Hospital Juárez, cuna de la cirugía y la neurocirugía. Apareció en: http://hospitaljuarezdemexico. blogspot.com/2019/10/el-hospital-juarez-cuna-de-la-cirugia-y. html?m=0.

3. Archivo histórico de la Facultad de Medicina de la Universidad Nacional Autónoma de México (AHFM), vol. 1, exp. 7.

4. Archivo histórico de la Facultad de Medicina de la Universidad Nacional Autónoma de México (AHFM), vol. 1, exp. 7.

5. Actualización deontológica de la profesión de médico cirujano. Círculo Médico Católico. Curia del Arzobispado de México. México, 1958.
6. Archivo histórico de la Facultad de Medicina de la Universidad Nacional Autónoma de México (AHFM), vol. 1, exp. 7.

7. Saldívar O, Jiménez X. Asambleas Nacionales de Cirujanos de México, A.C. Rev Hosp Jua Mex. 2012;79:280-287.

8. Acosta GR. Cien años de historia de la Revista del Hospital Juárez de México. Rev Hosp Jua Mex. 2012;79:288-298.

9. Revista de Cirugía. Órgano de la Sociedad de Cirugía. Año V, no. 9, septiembre de 1934.

10. Anónimo. Revista Universidad de México. "Exámenes médicos". ( $2^{\text {a }}$ columna) Apareció en: https://www.revistadelauniversidad.mx/ articles-files/3e711864-07f8-453b-acb6-b8ee9a6ac03e.

11. Fernández del Castillo F, Ardizana L. Cirugía y Cirujanos. Índice cronológico, de materias y autores. México, 1987. 